\title{
Science and style
}

\author{
Giacomo Prando explains how his experience as an editor helped him broaden his horizons as a scientist.
}

$\mathrm{T}$ hose working in a laboratory know the efforts needed to give an experimental answer to a scientific question. The path includes solving difficult conceptual issues - formulating the question in the first instance and designing proper physical systems and experimental set-ups apt to answer that question - as well as dealing with more technical aspects and problems of the implementation of the experiment. The circle is finally squared by sorting out the results and then examining their possible interpretations to find the most reasonable and satisfying one.

The final process of writing about the results is highly educational in my opinion. The necessity of describing the whole path in detail makes the comprehension of the results much clearer and more robust, leading to new insights - often from a perspective unseen before. In my view, the process has many similarities to preparing a lesson, which can be as educational for the teacher as it is for the students. So I realize that in the past, while writing my $\mathrm{PhD}$ thesis, a paper or an experimental report, I was explaining clearly to myself what I had done and what I had found, and in doing so my understanding naturally improved. As a result, I have often published logically ordered but very long, detailed and technical works, implicitly assuming that they would have been as useful to the readers as they were to me.

The truth is that, despite the good intentions, that is perhaps not the best way of communicating with other researchers and of making the most of the efforts and the time invested. Perhaps scientists working in the same field would easily understand and judge the significance of technical reports. But how many researchers do actually belong to the same field as science gets more and more specialized? The history of science shows that borrowing concepts from other fields is an extremely fertile exercise, so reaching out to wide audiences is a capital task for every scientist. How to do this in an effective and impactful way, possibly helping to foster and reinvigorate the scientific culture and attitude among the general public as well? This latter aspect is particularly relevant at this point in time, with scientific results too often being belittled - and scientists discredited - at different levels of society.

With these questions in mind I decided, after four years of post-doctoral work, that it was time for me to train in the best practice of scientific writing in a place where these issues are addressed as an explicit mission. I then proposed a short secondment to Nature Research Journals within the application for a Marie Skłodowska-Curie fellowship with the goal of improving my skills in science communication. Soon after, I applied for a fixed-term editorial position at Nature Nanotechnology, where I started working in March 2016. The 20 months I spent there were a much longer period than the originally planned 6 months, and they were more fruitful than I could have imagined.

\section{Looking well beyond the comfort-zone of my research field in such an intensive way was immensely instructive.}

As I started as an editor I realized that it was not just writing that I would have to deal with. I was immediately submerged by manuscripts reporting fascinating results across a wide range of topics, often quite distant from my own scientific background. The great responsibility of making an editorial decision on each manuscript - whether to proceed with publication, subject to further advice by expert referees, or not - made the feeling even more overwhelming. I had to read a vast amount of recent literature in different fields, aiming at the fairest possible contextualization of every manuscript within the current state of the art. Incidentally, I also found myself writing a lot of notes about each manuscript and, again, I realized how helpful this process was to reach a convincing decision. As some authors will know, these steps took me time, but I feel quite safe stating that my decisions were always as thoughtful and balanced as they could be.

In the process, I naturally found myself in the classroom again, expanding my knowledge broadly - rather than narrowly — as necessarily required in active research. In this sense, the multi- and interdisciplinarity of the topics covered by Nature Nanotechnology were a great advantage, and I am sure many will agree (citing from an author's correspondence: "the catholic tastes of the journal seem, to us, one of its great virtues").

The writing did come in at some point, especially with those manuscripts that I selected for further editorial consideration and which, eventually, got through the peer-review process and were accepted for publication. I admit that I felt so involved in the process that I considered those works as if they were partly mine, a feeling shared by other colleagues towards the manuscripts they handled. I wanted to see the results discussed in those papers, which by the time of the formal acceptance I knew almost by heart, conveyed to the reader in the most effective way. At this stage, editing a text far from my own scientific expertise placed me in the best possible position to help express those concepts to a non-specialist. This is a very useful and insightful exercise that, in my opinion, every PhD student and researcher should become accustomed to, even before attempting to write their own paper.

I was often asked if I was worried about interrupting my academic career to become an editor, and I can only defend my choice strongly. Looking well beyond the comfort-zone of my research field in such an intensive way was immensely instructive. And, now that I am back in academia, I am already working to give an active scientific continuity to that experience by expanding into fields that I had not explored before. Thus, in answer to the other classic question I was often asked - and that I confess used to irritate me - "so, how is life outside science?", I can once more say "I would not know, I have never really left science".

\section{Giacomo Prando \\ Department of Physics, University of Pavia, Pavia, Italy. e-mail: giacomo.prando@unipv.it}

Published online: 10 April 2018 https://doi.org/10.1038/s41565-018-0114-2 